\title{
Turing Instability in the Solid State: Void Lattices in Irradiated Metals
}

\author{
M. W. Noble \\ Department of Materials, University of Oxford, Oxford OXI 3PH, United Kingdom \\ M. R. Tonks \\ Department of Materials Science and Engineering, University of Florida, \\ 549 Gale Lemerand Drive, Gainesville, Florida 32611, USA \\ S. P. Fitzgerald $\odot^{*}$ \\ Department of Applied Mathematics, University of Leeds, Leeds LS2 9JT, United Kingdom
}

(Received 5 April 2019; revised manuscript received 4 October 2019; accepted 7 April 2020; published 20 April 2020)

\begin{abstract}
Turing (or double-diffusive) instabilities describe pattern formation in reaction-diffusion systems, and were proposed in 1952 as a potential mechanism behind pattern formation in nature, such as leopard spots and zebra stripes. Because the mechanism requires the reacting species to have significantly different diffusion rates, only a few liquid phase chemical reaction systems exhibiting the phenomenon have been discovered. In solids the situation is markedly different, since species such as impurities or other defects typically have mobilities $\propto \exp \left(-E / k_{B} T\right)$, where $E$ is the migration barrier and $T$ is the temperature. This often leads to mobilities differing by several orders of magnitude. Here, we use a simple, minimal model to show that an important class of emergent patterns in solids, namely void superlattices in irradiated metals, could also be explained by the Turing mechanism. Analytical results are confirmed by phase field simulations. The model (Cahn-Hilliard equations for interstitial and vacancy concentrations, coupled by generation and annihilation terms) is generic, and the mechanism could also be responsible for the patterns and structure observed in many solid state systems.
\end{abstract}

DOI: 10.1103/PhysRevLett.124.167401

Introduction.-Patterns formed by Turing instabilities [1] arise in reaction-diffusion systems due to the competition between diffusion and nonlinear reaction terms. Counterintuitively, a uniform solution for reactant concentrations (known as a base state), stable in the absence of diffusion, can become unstable to the emergence of patterns and ordering once diffusion is switched on. This runs counter to the standard picture of diffusion as a smoothing influence, and is interesting to study from a nonequilibrium physics point of view. Some time after Turing's original prediction, chemical systems were discovered that exhibited the effect, though they remain rare since the Turing model typically requires the reacting species to diffuse at significantly different rates-unusual in liquid phase chemical systems [2]. In the solid state, however, different species' diffusion rates generically differ by many orders of magnitude, since they are usually governed by nonlinear Arrhenius escape rates $\propto \exp \left(-E / k_{B} T\right)$, where the migration barrier $E$ can vary from fractions of to several electronvolts. We note that crowdion defects in bodycentered-cubic (bcc) metals have migration barriers too low for the Arrhenius formula to apply, and their diffusion rates are linear in temperature $[3,4]$.

An intriguing and technologically important example of solid state pattern formation is void and gas bubble superlattice formation in irradiated metals. First observed in the 1970s [5-7], the voids generated by the agglomeration of the radiation-induced vacancies can form an ordered superlattice under certain conditions. This runs counter to the more intuitive picture of Ostwald ripening, where large voids grow at the expense of smaller ones. Also, noble gases formed in fission reactors (e.g., $\mathrm{Kr}, \mathrm{Xe}$ ) generally have very low solubility in metals, and hence segregate to regions of high tensile strain. At grain boundaries, this leads to embrittlement, and accelerated mechanical failure. Engineering a stable bubble lattice (formed of voids filled with gas atoms) potentially offers a way to sequester the gas atoms safely away from grain boundaries and extend the life of reactor materials [8]. Superlattices are most often observed within a temperature window of $0.2-0.4$ of the melting point [9], and often mimic the lattice symmetry of the underlying crystal, though with a spacing tens or hundreds of times larger; see [10] for a thorough review. These lattices form over minutes and hours, meaning molecular dynamics simulations cannot hope to directly capture the processes at work.

Various competing mechanisms for superlattice formation have been proposed, including elastic interactions between voids, isomorphic decomposition, phase instability, interstitial dislocation loop punching and anisotropic 
interstitial diffusion [11-16]. Here, we propose an alternative mechanism, and argue that void lattices could emerge as a Turing instability, where diffusion itself destabilizes the uniform base states which solve the steady-state, diffusionless equations of motion. While some or all of the mechanisms above may play a role in the details of the superlattice formation, we show all that is actually required is a region in which local vacancy and interstitial concentration, generation, and annihilation rates satisfy a specific relation, and vacancy and interstitial diffusion rates that are sufficiently different. Ours is the simplest possible model that can capture the diffusion of two reacting species, with like species tending to cluster. It is nondimensionalized and is a gross idealization, and neglects many important features of real crystal systems, in particular the anisotropic nature of self-interstitial diffusion and the elastic interactions between species. Nevertheless, it is sufficient to predict the formation and length scale of ordered patterns, as we show below. Our purpose here is to present a minimal and general model, which may be applied to many different systems, rather than to focus on the details of specific materials. A systematic study dealing with particular metals and irradiation conditions will be published elsewhere.

In the next section, we apply Turing's linearized analysis to the pair of coupled equations governing the diffusing defects, and extract analytical conditions for the system to support a superlattice of a given wave number. We then perform fully nonlinear phase field simulations to investigate the system behavior at longer times, confirming that the superlattice wave number predicted by the linear analysis is indeed realised in the full system.

The model.-All crystalline materials have equilibrium defect concentrations $c_{\mathrm{eq}}$ at finite temperature $T$, depending on their formation energies $E_{f}$ via $c_{\mathrm{eq}} \propto \exp \left(-E_{f} / k_{B} T\right)$. Nonequilibrium concentrations can be dynamically generated in the crystal, e.g., when the stress relief afforded by a climbing dislocation outweighs the cost in energy to create a defect [17], or when prismatic loops are "punched" from the surface of overpressurized gas bubbles [18]. In particular, concentrations much greater than typical $c_{\text {eq }}$ values can be generated by neutron or ion irradiation, and it is these conditions under which void lattice formation has been observed. In what follows, we model the evolution of the defect populations using their continuum concentration fields, $v(\boldsymbol{x}, t)$ and $s(\boldsymbol{x}, t)$ for vacancies and self-interstitials, respectively. A phase field model [19-23] for their evolution leads to Cahn-Hilliard equations [24], with additional terms corresponding to creation and annihilation. Defects are assumed to be uniformly created at a certain rate $c$ throughout the material by displacement damage due to irradiation, and annihilation between vacancies and interstitials occurs at a rate $-a s(\boldsymbol{x}, t) v(\boldsymbol{x}, t)$, according to the law of mass action. Voids are identified as regions where the vacancy concentration $v$ is near 1 .

$$
\begin{aligned}
& \dot{s}=M_{s} \nabla^{2}\left(\frac{\delta F[s, v]}{\delta s}\right)+c-a s v \\
& \dot{v}=M_{v} \nabla^{2}\left(\frac{\delta F[s, v]}{\delta v}\right)+c-a s v .
\end{aligned}
$$

The terms in brackets are functional derivatives of the following simple double-well free energy $F[s, v]$ with respect to $s$ and $v$ :

$F=\int_{V}\left[s^{2}(1-s)^{2}+\frac{\gamma_{s}}{2}|\nabla s|^{2}+v^{2}(1-v)^{2}+\frac{\gamma_{v}}{2}|\nabla v|^{2}\right] d V$.

The quartic bulk free energy terms have minima when the concentrations $s$ and $v$ are 0 or 1 , encouraging the formation of voids and clusters. $M_{s}$ and $M_{v}$ are the atomic mobilities, which are proportional to the diffusivities $D_{s}$, $D_{v}$, with $M_{s} \gg M_{v}$ in metals, and the $\gamma$ 's are proportional to the square of the effective interface size between solid and void and cluster regions. We stress that all these parameters take effective values. Since superlattice formation takes place on a timescale of hours, the underlying atomic processes will be averaged over many realizations. For example, the annihilation rate $a$ does not represent the probability of mutual annihilation when a vacancy and selfinterstitial atom meet, but rather the fraction of defects which annihilate over a representative region in a representative time interval.

The explicit form of the equations is

$\dot{s}=M_{s} \nabla^{2}\left[2 s(s-1)(2 s-1)-\gamma_{s} \nabla^{2} s\right]+c-a s v ;$
$\dot{v}=M_{v} \nabla^{2}\left[2 v(v-1)(2 v-1)-\gamma_{v} \nabla^{2} v\right]+c-a v s$.

These equations conserve the number of defects during evolution (apart from the explicit creation and annihilation terms), in contrast with the coupled rate equation model [25] explored in Ref. [10], which involves only two spatial derivatives. Note that the defects do not interact until they meet and react: this is not a Fokker-Planck model of diffusion in a position-dependent potential, but rather a reaction-diffusion one. In the absence of the creation and annihilation terms, the two concentrations would decouple, and standard coarsening behavior would be expected (Ostwald ripening [18]). Below, we demonstrate that for certain ranges of parameter values, the Turing mechanism leads instead to long-range ordering, and the emergence of remarkably stable void lattices.

Analytical results.-We now follow the analysis due to Turing, and linearize the system about a so-called base state $\bar{s}, \bar{v}$ which satisfies the static equations, Eqs. (3) with all spatial and temporal derivatives set to zero:

$$
s(\boldsymbol{x})=\bar{s}+S(\boldsymbol{x}) ; \quad v(\boldsymbol{x})=\bar{v}+V(\boldsymbol{x}) ; c-a \bar{s} \bar{v}=0 .
$$


This imposes a relation between the uniform base states and the creation and annihilation rates. Seeking solutions of the form $(S, V) \equiv \boldsymbol{S}=\boldsymbol{S}_{0} \exp [\lambda t+i \boldsymbol{q} \cdot \boldsymbol{x}]$ leads to the eigenvalue equation $\lambda S_{0}=A_{q} S_{0}$, with

$\boldsymbol{A}_{q}=\left(\begin{array}{cc}-M_{s}\left(q^{2} g_{s}+\gamma_{s} q^{4}\right)-a \bar{v} & -a \bar{s} \\ -a \bar{v} & -M_{v}\left(q^{2} g_{v}+\gamma_{v} q^{4}\right)-a \bar{s}\end{array}\right)$,

where $q=|\boldsymbol{q}|=\left(q_{x}^{2}+q_{y}^{2}\right)^{1 / 2}$ in $2 \mathrm{D}, g_{s}=2[6 \bar{s}(\bar{s}-1)+1]$, and $g_{v}=2[6 \bar{v}(\bar{v}-1)+1]$. The eigenvalues $\lambda$ are given by the two solutions to $\operatorname{det}\left(\boldsymbol{A}_{\boldsymbol{q}}-\lambda \boldsymbol{I}\right)=0$. If both solutions for $\lambda(q)$ are negative, the solution decays in time, and hence the base state is stable to perturbations of wave number $\boldsymbol{q}$. A Turing instability arises when a base state is stable for $M_{s}=0=M_{v}$, but becomes unstable when it is perturbed by a certain wave number $\boldsymbol{q}$. The growing solution then leads to periodic patterns with wave number $\boldsymbol{q}$.

When $q=0, \lambda=0$ or $-a(\bar{s}+\bar{v})$, so for all base states, no unstable $(\lambda>0)$ pattern-forming mode is possible without diffusion. When diffusion is switched on, one or both eigenvalues are pushed above zero when either $\operatorname{tr} \boldsymbol{A}_{\boldsymbol{q}}>0$ and $\left(\operatorname{tr} \boldsymbol{A}_{\boldsymbol{q}}\right)^{2}-4 \operatorname{det} \boldsymbol{A}_{\boldsymbol{q}}>0$, or $\operatorname{tr} \boldsymbol{A}_{\boldsymbol{q}}<0$ and $\operatorname{det} \boldsymbol{A}_{\boldsymbol{q}}<0$. A sufficient (but not necessary) condition for the Turing instability is hence $\operatorname{det} \boldsymbol{A}_{\boldsymbol{q}}<0$. Assuming $\gamma_{s}=\gamma_{v}$, and working in units where $\gamma_{s}=\gamma_{v}=1$ leads to

$$
\begin{aligned}
\operatorname{det} \boldsymbol{A}_{q}= & M_{v} M_{s} q^{8}+M_{v} M_{s}\left(g_{s}+g_{v}\right) q^{6} \\
& +\left[M_{v} M_{s} g_{s} g_{v}+a\left(M_{v} \bar{v}+M_{s} \bar{s}\right)\right] q^{4} \\
& +a\left(M_{v} \bar{v} g_{v}+M_{s} \bar{s} g_{s}\right) q^{2},
\end{aligned}
$$

a quartic in $q^{2}$, passing through $q^{2}=0$ (reflecting the conservation of vacancies and interstitials). Positive values of $q^{2}$ that lead to a negative value of $\operatorname{det} \boldsymbol{A}_{q}$ correspond to a pattern with wave number $\boldsymbol{q} . q>2 \pi$ is not physically interesting, since it corresponds to patterns of wavelength less than the interface width. Also, $q \rightarrow 0$ corresponds to complete decomposition into void and undefected crystal, the standard coarsening behavior that would be expected without the $c$ and $a$ terms [see Supplemental Material (SM) [26]].

Since the equation for the determinant is effectively a cubic, it can be solved analytically, and the value of the superlattice spacing $\Lambda$ can be extracted as a function of the input parameters. This is given by $\Lambda=2 \pi / \sqrt{Q_{*} / 2}$, where $Q_{*}$ is the largest root of $d\left(\operatorname{det} \boldsymbol{A}_{q}\right) / d Q=0$ (see Fig. 1).

$M_{s} \gg M_{v}$ means that the interstitials generated during a cascade diffuse away faster than the vacancies, typically leading to a "halo" of interstitials surrounding a region of high vacancy density. Setting $\bar{v}=0.25$ and $\bar{s}=0.01$ to reflect an example of this results in the determinant shown in Fig. 1, for several values of the mobility ratio. When $M_{s}=M_{v}$, the determinant barely dips below zero, but as

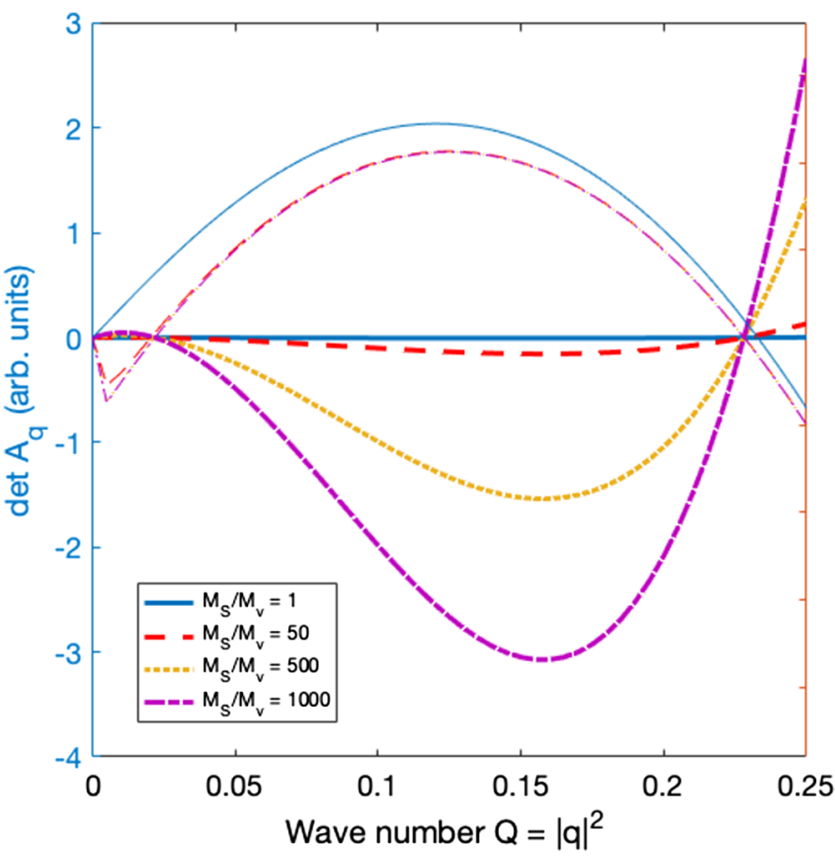

FIG. 1. Deepening instability as $M_{s} / M_{v} \rightarrow 1,50,500,1000$. $\bar{s}=0.01, \bar{v}=0.25, c=a \bar{s} \bar{v} . a=0.5$ here but the general behavior is not particularly sensitive to its value. Faint lines show the maximum eigenvalue, largest near $Q=0.12$, corresponding to the wave number with the fastest-growing instability. This is the wave number that the emergent pattern adopts, as confirmed by our numerical simulations.

the ratio $M_{s} / M_{v}$ increases up to the value of 1000 typical for bcc metals, the instability deepens. The maximum, most unstable, eigenvalue occurs for a wave number $q$ of approximately $\sqrt{0.12}$ for these parameters, corresponding to a pattern period $\Lambda=2 \pi /(q / \sqrt{2})$ of about 25 times the interface width, or around 100 spacings of the underlying crystal lattice, if we take the interface to be four crystal lattice spacings in width (again, this is an effective quantity, chosen to appropriately balance the bulk and interface terms in the free energy, and need not correspond precisely to the size of the physical interface at the void surface). This is consistent with experimentally observed void lattices. The $M_{s}=M_{v}$ system has a positive eigenvalue across the range of wave numbers, including $q=0$. This means that it is unstable to complete decomposition rather than the finite band of wave numbers characteristic of a Turing instability (see below).

The above values for $\bar{v}$ and $\bar{s}$ represent a reasonable example, but in any irradiated crystal, different regions will have different values. The conditions for instability are not particularly restrictive, however. According to Descartes's rule of signs, a cubic has two positive roots (i.e., takes a form similar to Fig. 1) when there are two sign changes between the successive terms in Eq. (6), and the discriminant is positive. Since the first coefficient is always positive, this means the last coefficient must be positive, 
and at least one of the second and third coefficients must be negative. For $\bar{s}=0.01$ and $\bar{v}=0.25$, the last term in Eq. (6) becomes positive when $M_{s} / M_{v}$ exceeds 3.32. For ratios less than this value, the determinant is negative as $q \rightarrow 0$, meaning that, unlike for the larger-ratio case, the system is unstable to complete decomposition-Ostwald ripening. In the limit of extremely different mobilities, where, say, $M_{s} \rightarrow \infty$, the interstitials diffuse instantaneously, and the field $s$ cannot support gradients. The two fields do not decouple entirely however, since the vacancy field $v$ still interacts with the average value of $s$. This leads to a modified evolution equation for $v$ that still supports pattern formation, and is equivalent in this limit to the Ohta-Kawasaki model [27,28] (see SM for further discussion [26]).

Phase field simulations.-The Turing analysis is based on linearization, and it is reasonable to ask whether the patterns remain once the nonlinearity becomes important, and the nascent regions of high vacancy concentration grow into voids. We used the open source Multiphysics Object Oriented Simulation Environment [29,30] to integrate Eqs. (3) numerically on a 2D domain (for computational efficiency; the Turing analysis depends only on $|\boldsymbol{q}|$ and is equally valid in 3D), using the finite element method with implicit time integration, starting from an initial condition randomized about $\bar{v}=0.25, \bar{s}=0.01$ (following a uniform distribution within the limits $\pm 1 \%$ ). $a=0.5$. The $300 \times 300$ domains were discretized using a $540 \times 540$ grid with periodic boundary conditions and ran until time $=30000$ (spatial units are scaled to the interface width, while time is measured in units corresponding to $M_{v}=1 ; M_{s} / M_{v}$ was varied from 1 to 1000). The initial condition for each field is a random spatial perturbation around constant values $\bar{s}, \bar{v}$, with no preexisting voids. Preexisting void populations dynamically rearrange themselves to form a similar lattice under certain conditions (see SM [26]). We tested different grid sizes and times with no effect on the results (see SM [26]).

The results are shown in Fig. 2(a). The initial condition is within the spinodal region for the vacancies, such that voids decompose with no discernable pattern. When $M_{s} / M_{v} \leq 2$, the voids evolve with no apparent ordering; however, when $M_{s} / M_{v} \geq 5$, ordering clearly emerges. This is consistent with the threshold value of $M_{s} / M_{v}=3.32$ predicted by the Turing analysis.

The superlattice spacing is approximately 25 units, confirming that the system selects the fastest-growing unstable mode as predicted by the analytical model. The lattice is hexagonal, which is the expected symmetry that minimizes the free energy for a given wave number; the equivalent in three dimensions is bcc [31].

Figures 2(b) and 2(c) show the number of voids and average void radius against time. Initially, voids decompose in the regions where the fluctuating initial vacancy concentration is high. For $M_{s} / M_{v}<3.32$, the standard picture of Ostwald ripening emerges, with large voids growing at the expense of smaller ones. As $M_{s} / M_{v}$ is increased, however, the number of voids stabilizes, and an ordered lattice emerges, as is clear from Fig. 2(a). The final, stable number of voids increases as $M_{s} / M_{v}$ increases, and the stable average void radius decreases, up to $M_{s} / M_{v}=100$, above which the stable number of voids and average void radius no longer change. This is true as $M_{s} \rightarrow \infty$ (see SM [26]). The slope on the log-log plot of the average void radius with time [Fig. 2(c)] is constant for ratios below
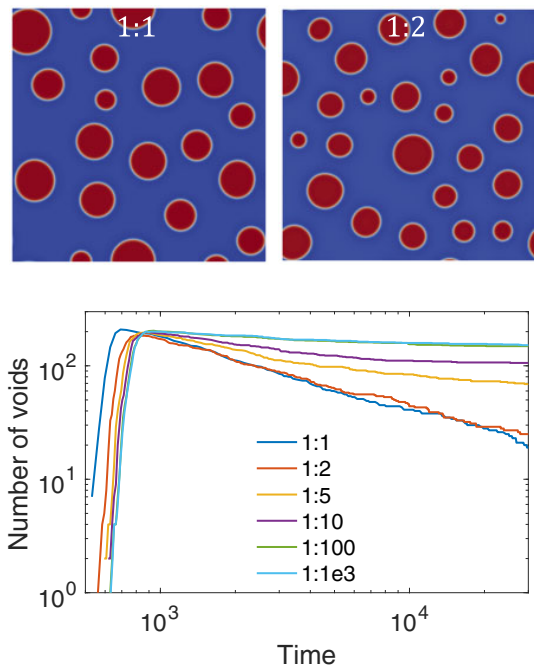

(b)

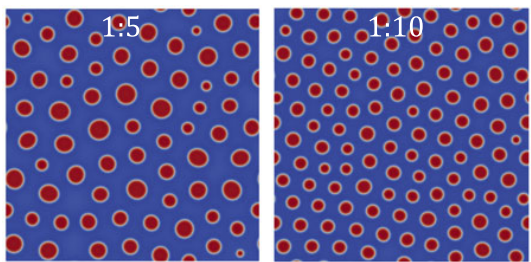

(a)

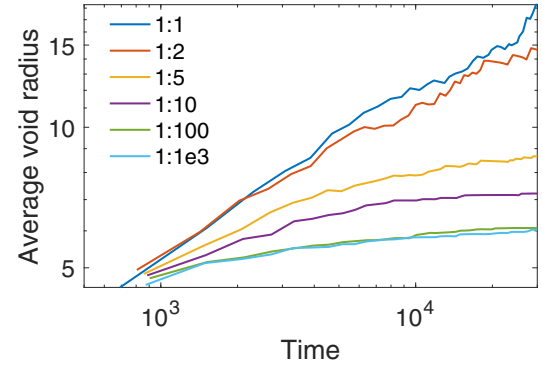

(c)
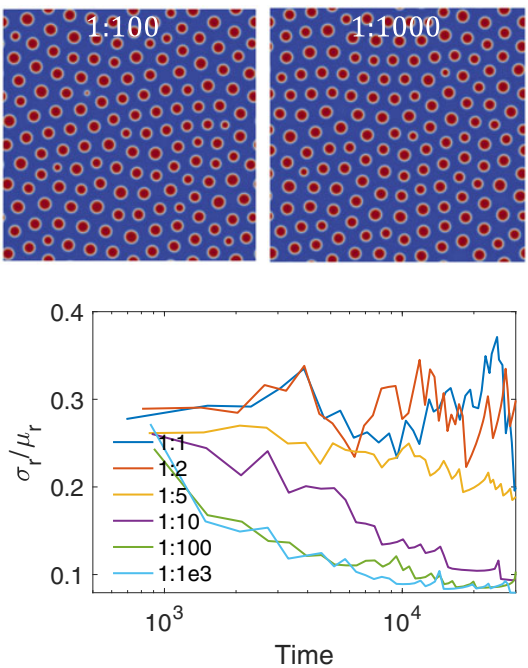

(d)

FIG. 2. (a) Phase field simulations of a 2D system governed by Eq. (3) at $t=30000$ for increasing values of the $M_{s} / M_{v}$ ratio. (b) void number, (c) mean void radius, and (d) spread in size (void radius standard deviation/mean, $\sigma_{r} / \mu_{r}$ ) vs time as $M_{s} / M_{v} \rightarrow 1,2,5,10,100$, 1000. The Turing pattern emerges when the mobility ratio grows above the critical value of 3.32 , and the lattice spacing is insensitive to the ratio. 
3.32, but decreases with time for higher ratios. This indicates atypical growth behavior that is not representative of standard Ostwald ripening, even with slow kinetics. This is also clearly indicated in the standard deviation plot [Fig. 2(d)]. The radius standard deviation to mean ratio is fairly constant for low mobility ratios (due to selfsimilarity), but when the ratios increase beyond five, the standard deviation goes down with time, which cannot be explained by slow kinetics.

We simulated the system under a variety of different initial conditions, including preexisting populations of voids of different sizes and distributions, and several different values for the creation term. In all cases with $M_{s} / M_{v}>3.32$, we found a stable void lattice (see SM [26]). Smaller voids on the lattice grow and larger voids shrink, and those not on lattice sites shrink until they disappear. Intriguingly, we also observed diffusion-driven migration of established voids to lattice locations, consistent with experimental observations [10]. This occurs in the absence of any advective term in the governing equations (1) and (3), and is purely due to the preferential diffusion of vacancies and interstitials so as to form the superlattice. This provides a mechanism for the fast migration of fairly large voids, which might intuitively be expected to be immobile.

Discussion.-We have shown that the simplest possible model for diffusing populations of vacancies and interstitials, subject to uniform creation and annihilation, supports void superlattice formation, even in the absence of refinements such as anisotropic interstitial diffusion and elastic interactions. The mechanism responsible for the ordering is the well-known Turing instability. This also offers a possible explanation for the observed temperature window for superlattice formation: the mechanism requires the mobilities of the vacancies and interstitials to differ significantly. The ratio $M_{s} / M_{v} \propto \exp \left[-\left(E_{\mathrm{mig}}^{\mathrm{int}}-E_{\mathrm{mig}}^{\mathrm{vac}}\right) / k_{B} T\right]$, and since $E_{\text {mig }}^{\text {int }}<E_{\text {mig }}^{\mathrm{vac}}$, it decreases at high temperature. At low temperatures, the vacancy diffusion rate is simply too slow for sufficient vacancies to cluster and form voids on experimental timescales.

This simple model is sufficient to qualitatively account for most of the phenomena observed in void lattice formation: the temperature window for formation, bcc superlattices appearing in bcc crystals, and hexagonal superlattices in hexagonal crystals [32]. Our model cannot predict fcc lattices (which have more than one inherent length scale). We have also observed the unexpected purely diffusion-driven migration of established voids to superlattice sites. The linearized Turing analysis predicts analytically the superlattice parameter in excellent agreement with fully nonlinear phase field simulations, even when the simulations are initialized with a preexisting population of randomly distributed voids. The remarkable robustness of stable superlattice formation, together with the simple and general nature of the model, suggests that Turing instabilities and their associated patterns could be generic in many solid state systems, where widely differing mobilities of different species are ubiquitous.

S.P.F. acknowledges discussions with Dr. P. Edmondson and Professor S. Donnelly, Professor A. Rucklidge, and Professor E. Knobloch, and financial support from the U.K. Engineering and Physical Sciences Research Council under Grant No. EP/ R005974/1. M. R. T. acknowledges faculty start-up support from the University of Florida.

*S.P.Fitzgerald@leeds.ac.uk

[1] A. M. Turing, Phil. Trans. R. Soc. B 237, 37 (1952).

[2] M. Cross and H. Greenside, Pattern Formation and Dynamics in Nonequilibrium Systems (Cambridge University Press, Cambridge, England, 2009).

[3] S. P. Fitzgerald and D. Nguyen-Manh, Phys. Rev. Lett. 101, 115504 (2008).

[4] T. D. Swinburne, S. L. Dudarev, S. P. Fitzgerald, M. R. Gilbert, and A. P. Sutton, Phys. Rev. B 87, 064108 (2013).

[5] J. Evans, Nature (London) 229, 403 (1971).

[6] P. Johnson and D. Mazey, Nature (London) 276, 595 (1978).

[7] V. Sikka and J. Moteff, J. Appl. Phys. 43, 4942 (1972).

[8] R. W. Harrison, G. Greaves, J. Hinks, and S. Donnelly, Sci. Rep. 7, 7724 (2017).

[9] A. M. Robinson, P. D. Edmondson, C. English, S. LozanoPerez, G. Greaves, J. Hinks, S. Donnelly, and C. R. Grovenor, Scr. Mater. 131, 108 (2017).

[10] N. Ghoniem, D. Walgraef, and S. Zinkle, J. Comput. Aided Mater. Des. 8, 1 (2001).

[11] K. Krishan, Radiat. Eff. 66, 121 (1982).

[12] V. Tewary and R. Bullough, J. Phys. F 2, L69 (1972).

[13] C. Woo and W. Frank, J. Nucl. Mater. 137, 7 (1985).

[14] A. Khachaturyan and V. Airapetyan, Phys. Status Solidi (a) 26, 61 (1974).

[15] J. Evans, J. Nucl. Mater. 132, 147 (1985).

[16] V. Dubinko, V. Slezov, A. Tur, and V. Yanovsky, Radiat. Eff. 100, 85 (1986).

[17] D. H. Thompson, E. Tarleton, S. G. Roberts, and S. P. Fitzgerald, Phys. Rev. Mater. 2, 080601 (2018).

[18] G. S. Was, Fundamentals of Radiation Materials Science: Metals and Alloys (Springer-Verlag, Berlin Heidelberg, 2016).

[19] N. Moelans, B. Blanpain, and P. Wollants, CALPHAD: Comput. Coupling Phase Diagrams Thermochem. 32, 268 (2008).

[20] L.-Q. Chen, Annu. Rev. Mater. Res. 32, 113 (2002).

[21] P. C. Millett and M. Tonks, Curr. Opin. Solid State Mater. Sci. 15, 125 (2011).

[22] Y. Li, S. Hu, X. Sun, and M. Stan, npj Comput. Mater. 3, 16 (2017).

[23] M. R. Tonks, A. Cheniour, and L. Aagesen, Comput. Mater. Sci. 147, 353 (2018).

[24] J. W. Cahn and J. E. Hilliard, J. Chem. Phys. 28, 258 (1958).

[25] R. Bullough, B. L. Eyre, and K. Krishan, Proc. R. Soc. A 346, 81 (1975). 
[26] See Supplemental Material at http://link.aps.org/ supplemental/10.1103/PhysRevLett.124.167401 for further simulations investigating the effect of changing various parameters, and an expanded discussion on the relation of the present model to the standard Cahn-Hilliard system.

[27] J. Kim, S. Lee, Y. Choi, S.-M. Lee, and D. Jeong, Math. Probl. Eng. 2016 (2016).

[28] T. Ohta and K. Kawasaki, Macromolecules 19, 2621 (1986).
[29] M. R. Tonks, D. Gaston, P. C. Millett, D. Andrs, and P. Talbot, Comput. Mater. Sci. 51, 20 (2012).

[30] D. Schwen, L. K. Aagesen, J. W. Peterson, and M. R. Tonks, Comput. Mater. Sci. 132, 36 (2017).

[31] H. Shoji, K. Yamada, D. Ueyama, and T. Ohta, Phys. Rev. E 75, 046212 (2007).

[32] D. Mazey and J. Evans, J. Nucl. Mater. 138, 16 (1986). 